\title{
Prevention of influenza in the general population
}

\author{
Joanne M. Langley, Marie E. Faughnan
}

ß See related article page 1169

Abstract

Background: Although all jurisdictions in Canada offer annual influenza immunization to people at high risk of complications, only Ontario has provided universal annual immunization of healthy adults and children. Use of chemotherapy (amantidine, neuraminidase inhibitors) to prevent influenza varies among provinces. We sought to systematically review the evidence for the prevention of influenza infection in the general population.

Methods: The interventions reviewed were influenza vaccination and prophylactic use of neuraminidase inhibitors. The health outcomes of interest were rates of laboratory-confirmed influenza infection, clinical definitions of influenzalike illness and work absenteeism. MEDLINE and Cochrane databases were searched for relevant articles published between 1966 and March 2003. Only randomized controlled trials (RCTs) were selected. Evidence was appraised using the methodology of the Canadian Task Force on Preventive Health Care.

Results: Eighteen trials involving more than 33000 healthy adults were identified that met the inclusion criteria; of these, 15 showed that influenza vaccination with either live-attenuated and inactivated vaccines was efficacious. Eleven trials were considered to be of "good" quality, and 7 were considered to be of "fair" quality. The relative risk reduction (RRR) associated with influenza immunization in adults ranged from $0 \%$ to $91 \%$. Fifteen RCTs involving more than 45000 healthy children aged 6 months to 19 years were identified, of which 9 were considered to contain "good" evidence and 6 "fair" evidence. Results from 12 of these trials showed protection against influenza. The RRR ranged from $0 \%$ to $93 \%$. There were 6 RCTs of "good" quality showing that neuraminidase inhibitors are effective in preventing influenza infection. Side effects from both influenza vaccination and neuraminidase inhibitor administration were mild.

Interpretation: There are numerous RCTs of good quality in large populations that have consistently shown that influenza vaccination, using inactivated or live-attenuated vaccines, is moderately effective in preventing influenza in the general population (healthy adults and children over 6 months of age). There is good evidence that neuraminidase inhibitor prophylaxis in contacts given within 36 to 48 hours of symptom onset of the household index case is effective; appropriate use of this prevention method requires access to rapid diagnostic methods. Decisions about introduction of routine immunization programs must take into account the cost and cost-effectiveness of a universal program and the burden of illness associated with influenza in each jurisdiction.

CMAJ 2004;171(10):1213-22

I nfluenza virus causes yearly epidemics of respiratory illness of varying severity worldwide in people of all ages, and it may be the most important cause of medically attended acute respiratory illness. ${ }^{1}$ In Canada influenza and pneumonia are the leading cause of death from infection and the sixth cause of death overall. ${ }^{2}$ Rates of complications and death from influenza are high among adults over 65 years of age and people with cardiac or pulmonary disease or chronic medical conditions, and annual influenza immunization in this population is associated with lower frequency of hospital admissions because of respiratory disease, congestive heart failure and death from any cause. ${ }^{3,4}$ Previously healthy young children are increasingly recognized as having hospital admission rates comparable to those among elderly people during influenza epidemics $^{5}$ and up to 12 -fold greater than rates among older children. ${ }^{6}$ Because influenza occurs yearly and because reinfections occur throughout the lifespan and affect up to $20 \%$ of the population each year, considerable attention has been directed to the prevention of influenza in healthy people. Although annual immunization programs are routinely offered to high-risk groups, only the province of Ontario routinely offers influenza immunization to healthy adults and children.

We performed a systematic review of the literature to answer the following question: how effective are the influenza vaccine and prophylactic neuraminidase inhibitor antiviral agents for the prevention of influenza in healthy adults and children?

\section{Methods}

We searched MEDLINE for relevant articles published between 1966 and March 2003 using the following search strategy for influenza vaccination trials: ("influenza vaccine" $[\mathrm{MeSH}]$ and "clinical trial" [publication type]) and (["human" (MeSH) or "ho- 
minidae" $(\mathrm{MeSH})]$ or "human $[\mathrm{MeSH}])$ and ("1996" [publication date] : "2003/03" [publication date]). The search strategy for trials on the effectiveness of neuraminidase inhibitor prophylaxis was as follows: ("neuraminidase/antagonists and inhibitors" $[\mathrm{MeSH}]$ and "clinical trial"[publication type]) and (["human" $(\mathrm{MeSH})$ or "hominidae" $(\mathrm{MeSH})]$ or "human" $[\mathrm{MeSH}])$ and ("1996" [publication date] : "2003/03" [publication date]) and (clinical trials or randomized clinical trials). The Cochrane Collaboration Library was also searched using the MeSH terms "influenza vaccine" and "neuraminidase" for these 2 searches respectively.

Our inclusion criteria for this review were (a) any randomized controlled trial (RCT) of influenza vaccines or neuraminidase inhibitors in humans and (b) an outcome measure of clinical efficacy against prevention of naturally occurring influenza in healthy people. A trial was considered randomized if the authors described the assignment of study drug or vaccine by random allocation or quasi-random allocation (e.g., alterna- tion, case record number), and it was considered controlled if there was a concurrent comparison group. Clinical efficacy measurement had to be determined by either a clinical definition of influenza or laboratory diagnosis; studies that measured only vaccine immunogenicity were excluded. Studies were also excluded if they were not in English or French or if they were targeted at high-risk groups, since recommendations already exist for these groups.

The MEDLINE search for influenza vaccine trials yielded 533 studies and the Cochrane search identified 4 reviews (Fig. 1). All of the studies identified through the Cochrane search were also found through the MEDLINE search. Review of the 533 titles led to the exclusion of 3 of the Cochrane reviews (ineligible patient population) and of individual studies for the following reasons: high-risk populations (149 studies), language other than French or English (56) and interventions other than influenza vaccine (e.g., education, compliance, Haemophilus influenzae vaccination) (81). Review of the abstracts of the re-

\section{Influenza vaccine trials}

Excluded $n=286$

- 149 studies of high-risk populations

- 56 studies published in language other than French or English

- 81 studies of interventions other than influenza vaccine

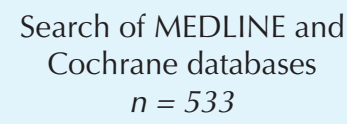

- 112 studies of immunogenicity

- 54 studies of compliance, delivery method, acceptability or adverse events; studies with different outcome measures

- 16 review articles

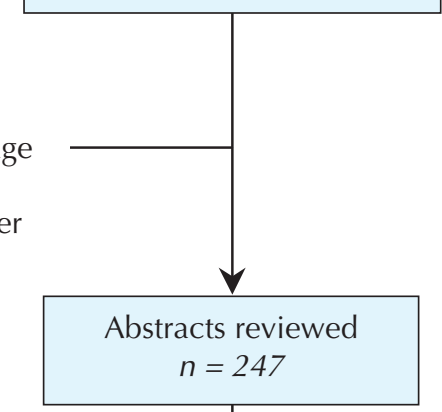

Antiviral trials

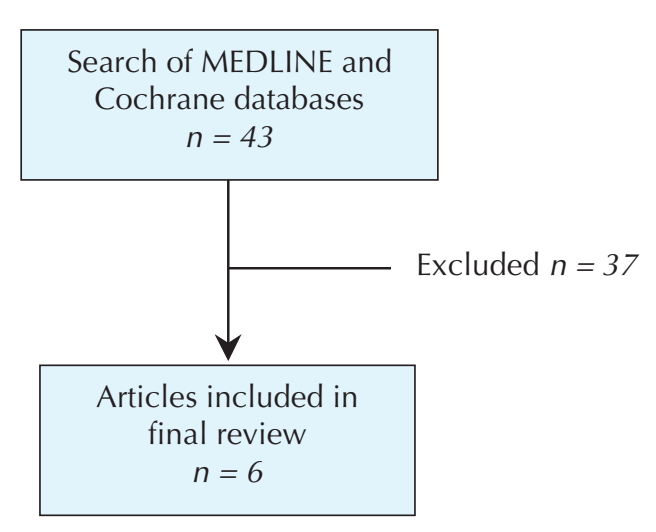

Methods reviewed $n=65$

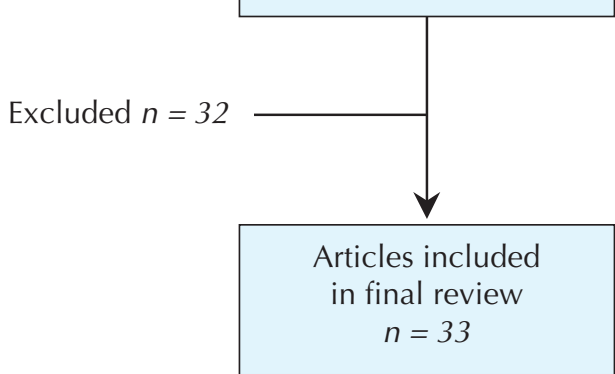

Fig. 1: Methodological steps for systematic review of influenza vaccine trials and antiviral therapy trials. 
maining 247 titles led to the exclusion of 182 studies. The most common reason was that the study outcome was vaccine immunogenicity or that it was a review article. Review of the methods of the 65 remaining studies identified 33 that satisfied our inclusion criteria. The antiviral search identified 43 studies in MEDLINE and 2 reviews in the Cochrane database (Fig. 1). Review of these articles identified 6 that satisfied our inclusion criteria. Some of the eligible trials that were identified through the MEDLINE search were also found in 1 of the Cochrane reviews.

The methods of the Canadian Task Force on Preventive Health Care were used to critically appraise the evidence from the included studies (Appendix 1). ${ }^{7}$ Quality ratings of individual studies - good, fair and poor - were determined on the basis of a set of operational parameters specific to randomized controlled trials developed with the US Preventive Services Task Force. ${ }^{8}$

\section{Results}

\section{Immunization of healthy adults}

Eighteen trials involving more than 33000 healthy adults were identified that met our criteria, and 15 of them demonstrated that influenza vaccination is efficacious (Table 1). Eleven trials with level I evidence were considered "good" quality. Seven trials with level I evidence were considered "fair" quality because of study characteristics that may have biased outcome ascertainment, including the presence $^{16,25}$ or possibility of unblinding ${ }^{13,14,24}$ of treatment assignment, lack of reliable outcome measurement ${ }^{13,18,25}$ or high loss of participants to follow-up. ${ }^{18,19}$ Eight trials used laboratory-confirmed influenza as an outcome measure, 9 used a clinical definition of influenza-like illness, and 1 trial used both outcomes.

Reflecting the variable annual attack rate of influenza, the incidence of laboratory-confirmed influenza in the control groups varied from 1.3 to 20 per 100 control subjects. In trials demonstrating a statistically significant difference, the RRR associated with influenza immunization ranged from $0 \%$ to $91 \%$. Both live-attenuated and inactivated vaccines were used with comparable efficacy; the results for these are combined where both were used within a trial.

The outcome measures used in these trials predominately captured acute influenza virus infection (e.g., laboratory-confirmed infection, influenza-like illness, febrile illness during peak influenza period, severe febrile illness, upper respiratory tract illness). Only 2 of 18 trials captured clinical outcomes related to influenza virus infections, such as hospital admissions and antibiotic use for respiratory infection. ${ }^{17,21}$ None of the trials evaluated secondary spread of influenza. Five studies used outcome measures related to work days lost that could potentially identify bacterial pneumonia as a complication of influenza virus infection. ${ }^{19-21,24,26}$ However, these studies did not use definitions for these outcomes that would allow the reader to accurately determine whether a bacterial pneumonia was pre- sent (e.g., chest radiograph, lower respiratory tract findings on examination). Event rates were higher in trials that used clinical definitions of influenza than in those that used laboratory confirmation. Event rates for laboratoryconfirmed influenza ranged from 1.3 to 20 per 100 control subjects and from 0.3 to 5.3 per 100 vaccinees. In trials using a clinical outcome measure, event rates ranged from 1.6 to 26 per 100 control subjects and from 2 to 27.9 per 100 vaccinees.

Six trials used outcome measures that captured the economic burden associated with respiratory illnesses not confirmed by laboratory methods to be influenza: lost work days because of illness, visits to a health care provider and use of prescription antibiotics and over-thecounter medications. These trials showed no reduc$\operatorname{tion}^{17,26}$ to modest reductions ${ }^{19-21,24}$ in lost time because of respiratory illness. A cost-benefit analysis of one of these influenza vaccination trials ${ }^{20}$ involving healthy working adults that used days of work missed, days at work but at reduced effectiveness and days with a visit to a health care provider because of an influenza-like symptom showed that vaccination (live-attenuated intranasal vaccine) reduced costs associated with all of these outcomes. ${ }^{27}$ The mean break-even cost for the vaccine and its administration was US\$43.07 using Monte Carlo analysis.

Adverse events were reported in all of but 5 trials. $11,13,16,25,26$ Among participants receiving injected vaccines, the most frequent side effects were local symptoms related to the injection site (e.g., pain, redness or induration). Statistically significant differences in these injection symptoms between vaccinees and placebo recipients were identified in several trials, with the highest incidence reported to be $64 \%$ among participants receiving inactivated injected vaccine, compared with $24 \%$ among placebo recipients. ${ }^{21}$ Various nonspecific complaints (e.g., tiredness, fever) were not more common among vaccine recipients than among control subjects. Recipients of live-attenuated vaccines were significantly more likely than placebo recipients to have a runny nose $(44 \% \text { v. } 26.6 \%)^{20}$ and sore throat $(15.6 \%-26.6 \%$ v. $6.6 \%-16.3 \%) .{ }^{10,12,20}$ Only 1 study related adverse events to subsequent medication use or lost work time; no difference between the vaccine and placebo groups was found. ${ }^{20}$

\section{Immunization of children}

Fifteen randomized controlled trials involving more than 45000 healthy children aged 6 months to 19 years were identified (Table 2); 9 trials with level I evidence were considered to be "good." Of the remaining 6 trials, 4 did not report blinded treatment assignment or outcome assessment $t^{29,37,38,42}$ and 4 did not have clear or uniform follow-up or application of outcome ascertainment. ${ }^{35,37,39,42}$ These 6 trials were therefore ranked as being of "fair" quality. Five trials used a clinical outcome mea- 
Table 1: Efficacy of influenza vaccination in healthy adults, by outcome (laboratory-confirmed [LC] influenza and clinical influenza)

\begin{tabular}{|c|c|c|c|c|c|c|c|c|c|c|c|}
\hline Study & $\begin{array}{l}\text { Type of } \\
\text { vaccine }\end{array}$ & $\begin{array}{l}\text { Follow- } \\
\text { up* }\end{array}$ & $\begin{array}{l}\text { No. of } \\
\text { subjects }\end{array}$ & Outcome & $\begin{array}{c}\text { Rate } \\
\text { per } 100 \\
\text { vaccinees }\end{array}$ & $\begin{array}{c}\text { Rate } \\
\text { per } 100 \\
\text { controls }\end{array}$ & $\begin{array}{c}\text { RRR } \\
(95 \% \mathrm{Cl}), \%\end{array}$ & $p$ value & NNT & $\begin{array}{c}\text { Level of } \\
\text { evidence† }\end{array}$ & $\begin{array}{l}\text { Quality of } \\
\text { evidence }\end{array}$ \\
\hline $\begin{array}{l}\text { Powers et al, } \\
1995^{9}\end{array}$ & $\begin{array}{l}\text { TRI-IA } \\
\text { v. placebo }\end{array}$ & 1 & 127 & LC influenza & 1.9 & 20 & $\begin{array}{c}91 \\
\text { (55 to 98) }\end{array}$ & 0.003 & 5.5 & I & Good \\
\hline \multirow[t]{4}{*}{$\begin{array}{l}\text { Edwards et } \\
\text { al, } 1994^{10}\end{array}$} & $\begin{array}{l}\text { BI-IA or TRI-IA } \\
\text { or BI-LA (IN) } \\
\text { v. placebo }\end{array}$ & $1-4$ & $\begin{array}{l}5210 \\
\text { (809 were } \\
\text { children) }\end{array}$ & $\begin{array}{l}\text { LC influenza, } \\
\text { yr } 1\end{array}$ & 0.68 & 3.2 & $\begin{array}{c}78 \\
\text { (58 to 89) }\end{array}$ & $<0.001$ & 52 & 1 & Good \\
\hline & & & & $\begin{array}{l}\text { LC influenza, } \\
\text { yr } 2\end{array}$ & 1.0 & 2.7 & $\begin{array}{c}63 \\
\text { (36 to } 79 \text { ) }\end{array}$ & 0.004 & & & \\
\hline & & & & $\begin{array}{l}\text { LC influenza, } \\
\text { yr } 3\end{array}$ & 0.49 & 2.8 & $\begin{array}{c}83 \\
\text { (66 to 91) }\end{array}$ & $<0.001$ & & & \\
\hline & & & & $\begin{array}{l}\text { LC influenza, } \\
\text { yr } 4\end{array}$ & 0.59 & 1.77 & $\begin{array}{c}66 \\
\text { (30 to } 84 \text { ) }\end{array}$ & 0.03 & & & \\
\hline \multirow[t]{2}{*}{$\begin{array}{l}\text { Kietel et al, } \\
1988^{11}\end{array}$} & $\begin{array}{l}\text { TRI-IA } \\
\text { v. placebo }\end{array}$ & $1-2$ & 1295 & $\begin{array}{l}\text { LC influenza, } \\
\text { yr } 1\end{array}$ & 5.3 & 9.3 & $\begin{array}{c}43 \\
(-3 \text { to } 43)\end{array}$ & 0.06 & 25 & I & Good \\
\hline & & & & $\begin{array}{l}\text { LC influenza, } \\
\text { yr } 2\end{array}$ & 3.7 & 7 & $\begin{array}{c}47 \\
(-2 \text { to } 72)\end{array}$ & 0.05 & 30 & & \\
\hline $\begin{array}{l}\text { Monto et al, } \\
1982^{12}\end{array}$ & $\begin{array}{l}\text { MONO-LA } \\
(\mathrm{IN}) \\
\text { v. placebo }\end{array}$ & 1 & 284 & LC influenza & 2.8 & 10.7 & $\begin{array}{c}74 \\
\text { (24 to } 91)\end{array}$ & 0.01 & 12.6 & 1 & Good \\
\hline $\begin{array}{l}\text { Hammond } \\
\text { et al, } 1978^{13}\end{array}$ & $\begin{array}{l}\text { TRI-IA } \\
\text { v. diphtheria- } \\
\text { tetanus }\end{array}$ & 1 & 225 & LC illness & 3.4 & 12.8 & $\begin{array}{c}73 \\
\text { (21 to } 91)\end{array}$ & 0.02 & 8.3 & 1 & Fair \\
\hline $\begin{array}{l}\text { Rytel et al, } \\
1977^{14}\end{array}$ & $\begin{array}{l}\text { BI-LA (IN) } \\
\text { v. placebo }\end{array}$ & 1 & 143 & $\begin{array}{l}\text { LC influenza } \\
\text { or influenza- } \\
\text { like illness }\end{array}$ & 3.6 & 11.3 & $\begin{array}{c}68 \\
(7 \text { to } 89)\end{array}$ & 0.04 & 25 & 1 & Fair \\
\hline $\begin{array}{l}\text { Mair et al, } \\
1974^{15}\end{array}$ & $\begin{array}{l}\text { MONO- } \\
\text { influenza A(IA) } \\
\text { v. MONO- } \\
\text { influenza B(IA) }\end{array}$ & 1 & 507 & LC influenza & 0.6 & 1.3 & $\begin{array}{c}50 \\
(-251 \text { to } 92)\end{array}$ & 0.4 & 100 & 1 & Good \\
\hline $\begin{array}{l}\text { Leibovitz et } \\
\text { al, } 1971^{16}\end{array}$ & $\begin{array}{l}\text { MONO v. } \\
\text { control }\end{array}$ & 1 & 9616 & LC influenza & 0.3 & 1.3 & $\begin{array}{c}77 \\
\text { (44 to } 91)\end{array}$ & $<0.001$ & 100 & 1 & Fair \\
\hline \multirow[t]{8}{*}{$\begin{array}{l}\text { Bridges et al, } \\
2000^{17}\end{array}$} & $\begin{array}{l}\text { TRI-IA } \\
\text { v. placebo }\end{array}$ & $1-2$ & 1184 & $\begin{array}{l}\text { LC influenza, } \\
\text { yr } 1\end{array}$ & 2.2 & 4.4 & $\begin{array}{c}46 \\
(-109 \text { to } 86)\end{array}$ & 0.33 & NA & 1 & Good \\
\hline & & & & $\begin{array}{l}\text { Hospital } \\
\text { admissions }\end{array}$ & 0.002 & 0.000 & NA & 0.5 & & & \\
\hline & & & & Antibiotic use & 0.057 & 0.070 & 18 & 0.09 & & & \\
\hline & & & 1191 & $\begin{array}{l}\text { LC influenza, } \\
\text { yr } 2\end{array}$ & 1.4 & 10.2 & $\begin{array}{c}86 \\
\text { (40 to 97) }\end{array}$ & 0.01 & 11.4 & & \\
\hline & & & & $\begin{array}{l}\text { Hospital } \\
\text { admissions }\end{array}$ & 0.0 & 0.0 & $\mathrm{NA}$ & $\mathrm{NA}$ & & & \\
\hline & & & & Antibiotic use & 0.041 & 0.055 & 25 & 0.047 & & & \\
\hline & & & 1184 & $\begin{array}{l}\text { Influenza-like } \\
\text { illness, yr } 1\end{array}$ & 27.9 & 23.8 & $\begin{array}{c}-17 \\
(-43 \text { to } 4)\end{array}$ & NS & $\mathrm{NA}$ & & \\
\hline & & & 1191 & $\begin{array}{l}\text { Influenza-like } \\
\text { illness, yr } 2\end{array}$ & 14 & 21 & $\begin{array}{c}34 \\
\text { (16 to 49) }\end{array}$ & 0.001 & 14 & & \\
\hline $\begin{array}{l}\text { Tannock et } \\
\text { al, } 1984^{18}\end{array}$ & $\begin{array}{l}\text { TRI-IA v. } \\
\text { placebo }\end{array}$ & 1 & 88 & $\begin{array}{l}\text { Respiratory } \\
\text { illness }\end{array}$ & 1.6 & 3.7 & $\begin{array}{c}42 \\
(-782 \text { to } 96)\end{array}$ & NA & NA & I & Fair \\
\hline \multirow[t]{2}{*}{$\begin{array}{l}\text { Mixeu et al, } \\
2002^{19}\end{array}$} & $\begin{array}{l}\text { TRI-IA v. } \\
\text { placebo }\end{array}$ & 1 & 593 & $\begin{array}{l}\text { Episodes of } \\
\text { influenza-like } \\
\text { illness }\end{array}$ & $\begin{array}{l}\text { 203/299 } \\
\text { people }\end{array}$ & $\begin{array}{l}121 / 294 \\
\text { people }\end{array}$ & & $<0.001$ & & 1 & Fair \\
\hline & & & & $\begin{array}{l}\text { Participants with } \\
\text { at least } 1 \text { episode } \\
\text { of influenza-like } \\
\text { illness }\end{array}$ & 33 & 29 & & NS & & & \\
\hline
\end{tabular}


Table 1 continued

\begin{tabular}{|c|c|c|c|c|c|c|c|c|c|c|c|}
\hline Study & $\begin{array}{l}\text { Type of } \\
\text { vaccine }\end{array}$ & $\begin{array}{l}\text { Follow- } \\
\text { up* }\end{array}$ & $\begin{array}{l}\text { No. of } \\
\text { subjects }\end{array}$ & Outcome & $\begin{array}{c}\text { Rate } \\
\text { per } 100 \\
\text { vaccinees }\end{array}$ & $\begin{array}{c}\text { Rate } \\
\text { per } 100 \\
\text { controls }\end{array}$ & $\begin{array}{c}\text { RRR } \\
(95 \% \mathrm{Cl}), \%\end{array}$ & $p$ value & NNT & $\begin{array}{l}\text { Level of } \\
\text { evidence }\end{array}$ & $\begin{array}{l}\text { Quality of } \\
\text { evidence }\end{array}$ \\
\hline & & & & $\begin{array}{l}\text { Participants with } \\
\text { at least } 1 \text { episode } \\
\text { of severe } \\
\text { influenza-like } \\
\text { illness }\end{array}$ & 15 & 9.8 & $\begin{array}{c}36 \\
\text { (19 to } 59)\end{array}$ & $<0.001$ & & & \\
\hline & & & & $\begin{array}{l}\text { Total work days } \\
\text { lost because of } \\
\text { influenza-like } \\
\text { illness }\end{array}$ & $102 \mathrm{~d}$ & $75 d$ & & $<0.05$ & & & \\
\hline & & & & $\begin{array}{l}\text { Employees with } \\
\text { work days lost } \\
\text { because of } \\
\text { influenza-like } \\
\text { illness }\end{array}$ & $\begin{array}{c}79 \\
\text { people }\end{array}$ & $\begin{array}{c}65 \\
\text { people }\end{array}$ & & NS & & & \\
\hline \multirow[t]{4}{*}{$\begin{array}{l}\text { Nichol et al, } \\
1999^{20}\end{array}$} & $\begin{array}{l}\text { TRI-LA (IN) v. } \\
\text { placebo }\end{array}$ & 1 & 4561 & $\begin{array}{l}\text { Febrile illness } \\
\text { during peak } \\
\text { influenza } \\
\text { period }\end{array}$ & 14.3 & 15.8 & $\begin{array}{c}10 \\
(-5 \text { to } 22)\end{array}$ & NS§ & 66 & $\begin{array}{l}\text { TRI-LA } \\
\text { (IN) v. } \\
\text { placeb } \\
\text { o }\end{array}$ & Good \\
\hline & & & & $\begin{array}{l}\text { Severe febrile } \\
\text { illness }\end{array}$ & 10.5 & 12.9 & $\begin{array}{c}18 \\
\text { (3 to } 31 \text { ) }\end{array}$ & 0.002 & 42 & & \\
\hline & & & & $\begin{array}{l}\text { Febrile upper } \\
\text { respiratory } \\
\text { tract illness }\end{array}$ & 8.6 & 11.4 & $\begin{array}{c}23 \\
\text { (7 to } 36)\end{array}$ & 0.001 & 36 & & \\
\hline & & & & $\begin{array}{l}\text { Sick days with } \\
\text { severe febrile } \\
\text { illness }\end{array}$ & 14.6 & 17.7 & $\begin{array}{c}17 \\
\text { (5 to } 28)\end{array}$ & 0.01 & 32 & & \\
\hline \multirow[t]{3}{*}{$\begin{array}{l}\text { Nichol et al, } \\
1995^{21}\end{array}$} & $\begin{array}{l}\text { TRI-IA } \\
\text { v. placebo }\end{array}$ & 1 & 849 & $\begin{array}{l}\text { Episodes of upper } \\
\text { respiratory tract } \\
\text { illness }\end{array}$ & $105 \ddagger$ & $\begin{array}{l}140 \neq \\
(69 \%)\end{array}$ & NA & 0.001 & 3 & I & Good \\
\hline & & & & $\begin{array}{l}\text { Sick days } \\
\text { because of upper } \\
\text { respiratory tract } \\
\text { illness }\end{array}$ & 70 & 122 & NA & 0.001 & 2 & & \\
\hline & & & & $\begin{array}{l}\text { Physician visits } \\
\text { because of upper } \\
\text { respiratory tract } \\
\text { illness }\end{array}$ & 31 & 55 & NA & 0.004 & 4 & & \\
\hline $\begin{array}{l}\text { Williams et } \\
\text { al, } 1973^{22}\end{array}$ & $\begin{array}{l}\text { MONO-IA, BI- } \\
\text { IA v. placebo }\end{array}$ & 1 & 13279 & $\begin{array}{l}\text { Influenza-like } \\
\text { illness }\end{array}$ & 6.5 & 8 & $\begin{array}{c}20 \\
(10 \text { to } 30)\end{array}$ & $<0.001$ & 50 & I & Good \\
\hline $\begin{array}{l}\text { Waldman et } \\
\text { al, } 1972^{23}\end{array}$ & $\begin{array}{l}\text { MONO-IA } \\
\text { (IN), BI-IA v. } \\
\text { placebo }\end{array}$ & 1 & 846 & $\begin{array}{l}\text { Influenza-like } \\
\text { illness }\end{array}$ & 9 & 20.4 & $\begin{array}{c}55 \\
\text { (31 to } 72 \text { ) }\end{array}$ & $<0.001$ & 8.7 & I & Good \\
\hline $\begin{array}{l}\text { Edmonson et } \\
\text { al, } 1970^{24}\end{array}$ & $\begin{array}{l}\text { BI-IA v. } \\
\text { MONO-IA }\end{array}$ & 1 & 1983 & $\begin{array}{l}\text { Work absence } \\
\text { because of } \\
\text { respiratory } \\
\text { illness }\end{array}$ & 22 & 26 & $\begin{array}{c}55 \\
(31 \text { to } 72)\end{array}$ & 0.025 & 25 & I & Fair \\
\hline $\begin{array}{l}\text { Eddy et al, } \\
1970^{25}\end{array}$ & $\begin{array}{l}\text { MONO-IA v. } \\
\text { placebo }\end{array}$ & 1 & 1758 & $\begin{array}{l}\text { Influenza-like } \\
\text { illness }\end{array}$ & 2 & 10 & $\begin{array}{c}80 \\
\text { (68 to } 88)\end{array}$ & $<0.001$ & 12.5 & I & Fair \\
\hline $\begin{array}{l}\text { Hobson et } \\
\text { al, } 1970^{26}\end{array}$ & $\begin{array}{l}\text { QUAD-IA v. } \\
\text { MONO-IA }\end{array}$ & 1 & 1601 & $\begin{array}{l}\text { Respiratory } \\
\text { illness causing } \\
\text { work absence }\end{array}$ & 2.9 & 1.6 & $\begin{array}{c}-84 \\
(-298 \text { to } 15)\end{array}$ & NA & NA & I & Good \\
\hline
\end{tabular}

Note: $\mathrm{RRR}=$ relative risk reduction, $\mathrm{Cl}=$ confidence interval, $\mathrm{NNT}=$ number needed to treat, $\mathrm{TRI}=$ trivalent, $\mathrm{IA}=$ inactivated, $\mathrm{BI}=$ bivalent, $\mathrm{LA}=\mathrm{live-attenuated}, \mathrm{IN}=$ intranasal, $\mathrm{MONO}=$ monovalent, QUAD = quadrivalent, $\mathrm{NA}=$ not applicable.

*Number of influenza seasons.

†See Appendix 1 for descriptions of the levels of evidence and quality ratings of trials.

¥Some of the rates exceed $100 \%$ of vaccinees, because many subjects had more than 1 URI or more than 1 day of absenteeism. Where possible, outcomes are reported by year in multiyear studies.

$\S$ Cochrane-Mantel-Haenszel. 
Table 2: Efficacy of influenza vaccination in healthy children, by outcome (laboratory-confirmed [LC] influenza and clinical influenza)

\begin{tabular}{|c|c|c|c|c|c|c|c|c|c|c|c|}
\hline $\begin{array}{l}\text { Study } \\
\text { (age group } \\
\text { studied) }\end{array}$ & Type of vaccine & $\begin{array}{l}\text { Follow- } \\
\text { up* }^{*}\end{array}$ & $\begin{array}{l}\text { No. of } \\
\text { subjects }\end{array}$ & Outcome & $\begin{array}{c}\text { Rate } \\
\text { per } 100 \\
\text { vaccinees }\end{array}$ & $\begin{array}{c}\text { Rate } \\
\text { per } 100 \\
\text { controls }\end{array}$ & $\begin{array}{c}\text { RRR } \\
(95 \% \mathrm{Cl}), \%\end{array}$ & $p$ value & NNT & $\begin{array}{c}\text { Level of } \\
\text { evidence } \dagger\end{array}$ & $\begin{array}{l}\text { Quality of } \\
\text { evidence }\end{array}$ \\
\hline $\begin{array}{l}\text { Neuzil et al, } \\
2001^{28} \\
(1-16 y r)\end{array}$ & $\begin{array}{l}\text { BI-IA, TRI-IA, TRI- } \\
\text { LA (IN) v. placebo } \\
\text { or MONO- } \\
\text { influenza B }\end{array}$ & $2-5$ & 791 & LC illness & 0.88 & 5.75 & $\begin{array}{c}85.9 \\
(72 \text { to } 93)\end{array}$ & $<0.001$ & 20 & I & Good \\
\hline $\begin{array}{l}\text { Hurwitz et } \\
\text { al, } 2000^{29} \\
(24-60 \mathrm{mo})\end{array}$ & $\begin{array}{l}\text { TRI-IA v. } \\
\text { hepatitis A }\end{array}$ & 1 & 145 & LC influenza & 28 & 51 & $\begin{array}{c}45 \\
(6 \text { to } 67)\end{array}$ & NS & 4 & I & Fair \\
\hline $\begin{array}{l}\text { Belshe et al, } \\
2000^{30} \\
(26-85 \mathrm{mo})\end{array}$ & $\begin{array}{l}\text { TRI-LA (IN) v. } \\
\text { placebo }\end{array}$ & 1 & 135 & LC influenza & 1.63 & 14.5 & $\begin{array}{c}87 \\
\text { (78 to } 87)\end{array}$ & $<0.001$ & 24 & I & Good \\
\hline $\begin{array}{l}\text { Belshe et al, } \\
1998^{31} \\
(15-71 \mathrm{mo})\end{array}$ & $\begin{array}{l}\text { TRI-LA (IN) v. } \\
\text { placebo }\end{array}$ & 1 & 160 & LC influenza & 1.31 & 17.9 & $\begin{array}{c}93 \\
\text { (88 to } 96)\end{array}$ & $<0.001$ & 6 & I & Good \\
\hline $\begin{array}{l}\text { Gruber et al, } \\
1996^{32} \\
(6-18 \mathrm{mo})\end{array}$ & $\begin{array}{l}\text { MONO-LA, } \\
\text { BI-LA (IN) v. } \\
\text { placebo }\end{array}$ & 1 & 182 & LC influenza & 6.45 & 24 & $\begin{array}{c}66 \\
\text { (18 to } 66)\end{array}$ & 0.009 & 8 & I & Good \\
\hline $\begin{array}{l}\text { Clover et al, } \\
1991^{33} \\
(3-18 \mathrm{yr})\end{array}$ & $\begin{array}{l}\text { LA (IN), TRI-IA } \\
\text { v. placebo }\end{array}$ & 1 & 192 & LC illness & 19 & 43.9 & $\begin{array}{c}56.5 \\
(31 \text { to } \\
72)\end{array}$ & $<0.001$ & 40 & I & Good \\
\hline $\begin{array}{l}\text { Gruber et al, } \\
1990^{34} \\
(3-19 \mathrm{yr})\end{array}$ & $\begin{array}{l}\text { BI-LA (IN), TRI- } \\
\text { IA v. placebo }\end{array}$ & 1 & 189 & LC influenza & 18.5 & 48 & $\begin{array}{c}61 \\
\text { (29 to } 62)\end{array}$ & $<0.001$ & 3 & I & Good \\
\hline $\begin{array}{l}\text { Feldman et } \\
\text { al, } 1985^{35} \\
(1-7 \mathrm{yr})\end{array}$ & $\begin{array}{l}\mathrm{LA}(\mathrm{IN}), \mathrm{BI}-\mathrm{IA} \text { v. } \\
\text { placebo }\end{array}$ & 1 & 111 & LC illness & 36 & 50 & $\begin{array}{c}-1 \\
(-36 \text { to } 25)\end{array}$ & 0.23 & 71 & I & Fair \\
\hline $\begin{array}{l}\text { Hoskins et } \\
\text { al, } 1973^{36} \\
(11-19 \mathrm{yr})\end{array}$ & $\begin{array}{l}\text { MONO-IA } \\
\text { influenza A v. } \\
\text { MONO-IA } \\
\text { influenza B }\end{array}$ & $1-2$ & 724 & LC influenza & 2.9 & 9.4 & $\begin{array}{c}70 \\
(41 \text { to } 70)\end{array}$ & $<0.001$ & 15 & I & Good \\
\hline $\begin{array}{l}\text { Wesselius- } \\
\text { de Casparius } \\
\text { et al, } 1972^{37} \\
(<5 \text { to }>10 \mathrm{yr})\end{array}$ & $\begin{array}{l}\text { MONO-IA v. } \\
\text { placebo }\end{array}$ & 1 & 374 & LC influenza & 9.8 & 16.2 & $\begin{array}{c}43 \\
(6 \text { to } 68)\end{array}$ & 0.05 & 109 & I & Fair \\
\hline $\begin{array}{l}\text { Colombo et } \\
\text { al, } 2001^{38} \\
(1-6 \mathrm{yr})\end{array}$ & $\begin{array}{l}\text { TRI-IA v. no } \\
\text { vaccine }\end{array}$ & 1 & 344 & $\begin{array}{l}\text { Influenza-like } \\
\text { illness; day-care } \\
\text { absenteeism }\end{array}$ & $\begin{array}{c}12.4 \\
0.5 \text { days }\end{array}$ & $\begin{array}{c}37.7 \\
2.3 \\
\text { days }\end{array}$ & $\begin{array}{c}67 \\
\text { (49 to } 79)\end{array}$ & $<0.001$ & 4 & I & Fair \\
\hline $\begin{array}{l}\text { Khan et al, } \\
1996^{39} \\
(9-12 \mathrm{yr})\end{array}$ & $\begin{array}{l}\text { TRI-IA, TRI-LA } \\
\text { v. placebo }\end{array}$ & 1 & 555 & $\begin{array}{l}\text { School absence } \\
\text { with doctor- } \\
\text { diagnosed acute } \\
\text { respiratory illness } \\
\text { or influenza }\end{array}$ & 0 & 3 & $\begin{array}{l}100 \\
(\mathrm{NA})\end{array}$ & $<0.05$ & 333 & I & Fair \\
\hline \multirow{2}{*}{$\begin{array}{l}\text { Rudenko et } \\
\text { al, } 1993^{40} \\
(7-14 \mathrm{yr})\end{array}$} & \multirow{2}{*}{$\begin{array}{l}\text { TRI-LA, BI- LA } \\
\text { (IN), BI-IA, TRI-IA } \\
\text { v. placebo }\end{array}$} & 2 & 12837 & $\begin{array}{l}\text { Respiratory } \\
\text { disease yr } 1\end{array}$ & 16.77 & 17 & $\begin{array}{c}2 \\
(-5 \text { to } 9)\end{array}$ & $<0.001$ & 333 & \multirow[t]{2}{*}{1} & \multirow[t]{2}{*}{ Good } \\
\hline & & & & $\begin{array}{l}\text { Respiratory } \\
\text { disease yr } 2\end{array}$ & 22.9 & 33 & $\begin{array}{c}30 \\
(26 \text { to } 34)\end{array}$ & $<0.001$ & 10 & & \\
\hline $\begin{array}{l}\text { Alexandrova } \\
\text { et al, } 1986^{41} \\
(3-16 \mathrm{yr})\end{array}$ & $\begin{array}{l}\text { BI-LA (IN) v. } \\
\text { placebo }\end{array}$ & 1 & 30000 & "Influenza" & 6 & 12 & $\begin{array}{c}52 \\
\text { (48 to } 56)\end{array}$ & $<0.001$ & 16 & I & Good \\
\hline $\begin{array}{l}\text { Maynard et } \\
\text { al, } 1968^{42} \\
(14-18 y r)\end{array}$ & $\begin{array}{l}\text { QUAD (IA) v. } \\
\text { MONO (IA) }\end{array}$ & 1 & 488 & $\begin{array}{l}\text { Influenza-like } \\
\text { illness }\end{array}$ & 49 & 55 & $\begin{array}{c}10 \\
(-9 \text { to } 25)\end{array}$ & NS & 16 & I & Fair \\
\hline
\end{tabular}

Note: See Table 1 for definitions of abbreviations. Where possible, outcomes are reported by year in multiyear studies.

†See Appendix 1 for descriptions of the levels of evidence and quality ratings of trials. 
sure for influenza, and 10 used laboratory confirmation. Consistent differences in efficacy between the inactivated and live-attenuated vaccines were not observed, and therefore the treatment arms are combined for the purposes of this review.

Twelve trials involving children demonstrated protection against clinical influenza, whether laboratoryconfirmed influenza or defined as influenza-like illness. In another 3 trials, benefit was not demonstrated. ${ }^{29,35,42}$ Influenza attack rates vary each influenza season, and this was reflected in disease incidence in the control groups of these studies, with laboratory-confirmed influenza in 5.75 to 51 per 100 control subjects and 0.88 to 36 per 100 vaccinees. Rates of influenza in studies using clinical outcome measures were 12 to 55 per 100 control subjects and 5.8 to 49 per 100 vaccinees. The highest efficacy rate $(93 \%$; $95 \%$ confidence interval $88 \%-96 \%$ ) was reported among children 15 to 71 months old receiving 1 or 2 doses of a liveattenuated, trivalent, intranasal influenza virus vaccine. Immunization did not prevent non-influenza respiratory tract illness.

Adverse events were reported in 12 of the 15 trials involving children. Both inactivated and live-attenuated vaccines were well tolerated, and no severe adverse reactions attributable to vaccine were observed in the studies reviewed. Recipients of a live-attenuated vaccine were significantly more likely than placebo recipients to have a runny nose 2 days after administration (27\% of children 26-85 months old v. $18 \%$ of control subjects), ${ }^{30}$ coryza
( $27.8 \%$ of children under 6 years old v. $17.7 \%$ of control subjects $\left.{ }^{28}\right)$ and fever $(6.5 \%$ of children $26-85$ months old had a mean temperature of $38.2^{\circ} \mathrm{C} \mathrm{v.} 1.6 \%$ of control subjects $\left.{ }^{30}\right)$. Inactivated vaccines were associated with induration at the injection site in children over 11 years of age in one study (14.2\% of vaccine recipients v. $4.4 \%$ of control subjects). ${ }^{28}$ Coryza and fever with live-attenuated vaccines occurred less frequently on reimmunization in subsequent seasons, with no difference observed between placebo and vaccine groups. ${ }^{30}$ Fever was more common among younger children than among older children regardless of vaccine type. ${ }^{28}$

\section{Neuraminidase inhibitor prophylaxis}

The efficacy of the neuraminidase inhibitors oseltamavir and zanamavir for influenza prophylaxis during community outbreaks has been evaluated in 6 randomized controlled trials since 1999; all of these had level I evidence and were of "good" quality (Table 3). The RRR ranged from $32 \%$ to $84 \%$, with influenza rates in the placebo groups ranging from $18 \%$ to $67 \%$ and in the prophylaxis groups from $3.6 \%$ to $38 \%$. Oseltamavir was evaluated in people over 12 years of age. Zanamavir was evaluated in people over 5 years of age.

Adverse events were reported in all 6 trials. Gastrointestinal side effects were more common in recipients of oseltamivir in the 3 trials of that agent $(9.3 \%$ v. $7.2 \%$ on placebo, ${ }^{45}$ and $13.1 \%$ [ 1 daily dose] and $14.6 \%$ [2 daily

Table 3: Efficacy of neuraminidase inhibitors compared with placebo for prophylaxis of laboratory-confirmed (LC) influenza

\begin{tabular}{|c|c|c|c|c|c|c|c|c|c|}
\hline \multirow[b]{2}{*}{ Study } & \multirow[b]{2}{*}{$\begin{array}{l}\text { Neuraminidase } \\
\text { inhibitor }\end{array}$} & \multirow[b]{2}{*}{ Study population } & \multicolumn{2}{|c|}{$\begin{array}{c}\text { Incidence of } \\
\text { LC influenza, \% }\end{array}$} & \multirow[b]{2}{*}{$\begin{array}{c}\text { RRR } \\
(95 \% \mathrm{Cl}), \%\end{array}$} & \multirow[b]{2}{*}{$p$ value* } & \multirow[b]{2}{*}{ NNT } & \multirow[b]{2}{*}{$\begin{array}{l}\text { Level of } \\
\text { evidence† }\end{array}$} & \multirow[b]{2}{*}{$\begin{array}{c}\text { Quality } \\
\text { of evidence } †\end{array}$} \\
\hline & & & $\begin{array}{l}\text { Treatment } \\
\text { group }\end{array}$ & $\begin{array}{l}\text { Placebo } \\
\text { group }\end{array}$ & & & & & \\
\hline $\begin{array}{l}\text { Hayden et al, } \\
1999^{43}\end{array}$ & $\begin{array}{l}\text { Oseltamavir } \\
\text { (oral) }\end{array}$ & $\begin{array}{l}33 \text { participants, } \\
\text { aged } 18-40 \mathrm{yr}\end{array}$ & 38 & 67 & $\begin{array}{c}43 \\
(-12 \text { to } 71)\end{array}$ & $0.11 \dagger$ & 34 & I & Good \\
\hline $\begin{array}{l}\text { Hayden et al, } \\
1999^{44}\end{array}$ & $\begin{array}{l}\text { Oseltamavir } \\
\text { (oral) }\end{array}$ & $\begin{array}{l}1559 \text { participants, } \\
\text { aged } 18-65 \mathrm{yr}\end{array}$ & 1.3 & 4.8 & $\begin{array}{c}74 \\
(53 \text { to } 88)\end{array}$ & $<0.001$ & 28 & I & Good \\
\hline $\begin{array}{l}\text { Welliver et al, } \\
2001^{45}\end{array}$ & $\begin{array}{l}\text { Oseltamavir } \\
\text { (oral) }\end{array}$ & $\begin{array}{l}377 \text { households, } \\
\text { participants aged } \\
>12 \mathrm{yr}\end{array}$ & 3.6 & 22.8 & $\begin{array}{c}84 \\
\text { (49 to } 95)\end{array}$ & $<0.001 \dagger$ & 5 & I & Good \\
\hline $\begin{array}{l}\text { Kaiser et al, } \\
2000^{46}\end{array}$ & $\begin{array}{l}\text { Zanamavir } \\
\text { (inhaled or } \\
\text { inhaled and } \\
\text { intranasal) }\end{array}$ & $\begin{array}{l}575 \text { participants, } \\
\text { aged } 13-65 \mathrm{yr}\end{array}$ & 12.7 & 18.7 & $\begin{array}{c}32 \\
(-7 \text { to } 32)\end{array}$ & NA & 16 & I & Good \\
\hline $\begin{array}{l}\text { Hayden et al, } \\
2000^{47}\end{array}$ & $\begin{array}{l}\text { Zanamavir } \\
\text { (inhaled) }\end{array}$ & $\begin{array}{l}337 \text { households, } \\
\text { participants aged } \\
>5 \mathrm{yr}\end{array}$ & 4 & 19 & $\begin{array}{c}78 \\
\text { (52 to } 78)\end{array}$ & $0.001 \ddagger$ & 6 & I & Good \\
\hline $\begin{array}{l}\text { Monto et al, } \\
2002^{48}\end{array}$ & $\begin{array}{l}\text { Zanamavir } \\
\text { (inhaled) }\end{array}$ & $\begin{array}{l}487 \text { households, } \\
\text { participants aged } \\
>5 \mathrm{yr}\end{array}$ & 4.1 & 19 & $\begin{array}{c}78 \\
\text { (58 to } 78)\end{array}$ & $<0.001 \dagger$ & 6 & I & Good \\
\hline
\end{tabular}

*Statistical test for proportions.

$+\chi^{2}$ test.

‡Exact test. 
doses] v. $7.1 \%$ on placebo). ${ }^{43-45}$ Nausea was the most common gastrointestinal adverse event. Adverse events were not significantly different in placebo and zanamavir prophylaxis arms.

\section{Interpretation}

This review indicates that effective influenza prevention interventions are available for healthy adults and children. Protection with influenza vaccine varied from moderate to high, with RRRs from $0 \%$ to $93 \%$. We also found that the neuraminidase inhibitors oseltamivir and zanamivir were effective in people over 12 years and over 5 years of age respectively. These drugs have the advantage of having fewer side effects than rimantadine and amantadine and are effective against influenza $\mathrm{A}$ and $\mathrm{B} .{ }^{49}$ In the studies included in this review, no serious adverse events were reported as a result of either the influenza vaccine or antiviral therapy.

The apparent variation in efficacy of the influenza vaccine over time and between trials is likely due to a number of factors, including vaccine immunogenicity and the degree of match between the vaccine strain chosen before the influenza season and the circulating virus strains. Influenza A virus changes over time as it propagates in humans, through an accumulation of point mutations and by genetic reassortment between viral strains. ${ }^{50}$ The World Health Organization recommends the composition of inactivated vaccines each year based on the occurrence of strains causing outbreaks that are reported from 110 surveillance centres in 83 countries and the availability of strains for production of vaccine. The "match" between predicted and circulating strains was $88 \%$ in a 10 -year period. ${ }^{51}$ Previous exposure to vaccine or natural infection may increase vaccine efficacy.

Influenza viruses cause illness in up to $20 \%$ of the general population each winter in nonpandemic years. Severe illness requiring hospital admission is most likely to occur in people with pre-existing lung or cardiac disease or chronic medical conditions and in people over 65 years of age. Immunization programs for these target groups, known to decrease rates of hospital admission and death, ${ }^{3}$ are in place across Canada, although uptake is incomplete. ${ }^{52}$ Influenza also causes a significant health burden in the general population in terms of hospital admissions, outpatient visits, sick leave and antimicrobial use. ${ }^{53}$ In particular, children under 2 years of age may have hospital admission rates of up to 112 per 100000 population. ${ }^{6,54}$ In an influenza pandemic, attack rates could exceed $30 \%$ of the general population. ${ }^{55}$

Recommendations for influenza vaccination have become more inclusive in recent years. The National Advisory Committee on Immunization suggests that "any individual who wishes to protect him/herself from influenza should be encouraged to receive the vaccine." ${ }^{56}$ The American Academy of Pediatrics ${ }^{57}$ and the Advisory Committee on Immunization Practices of the US Centers for Disease Control and Prevention ${ }^{58}$ recently revised its recommendations to include vaccination of children aged 6-23 months, and contacts of infants aged 0-23 months. Whereas Canada recommends immunization of people over 65 years of age, the US Centers for Disease Control and Prevention recommends the inclusion of people over 50 years, because of the increased incidence of high-risk conditions in that age group..$^{53}$ To date, Ontario is the only jurisdiction in Canada to introduce a universal influenza immunization program. ${ }^{59,60}$

The goal of our review was to determine the efficacy of the influenza vaccine and neuraminidase inhibitors, not to determine the efficacy of a universal immunization program. With such a program, considerations that must be weighed against the potential benefits (preventing illness and death in high-risk groups, and decreasing economic loss associated with absenteeism at work, visits to health care providers and antibiotic use) include the economic costs of vaccine and program delivery, vaccine procurement for large populations, the need to immunize large populations in a short period each year and public acceptability. There is some evidence that universal influenza immunization of school children is associated with a reduction in excess winter deaths in the general population. ${ }^{61}$ Now in its fourth year, the universal influenza program in Ontario may help to further clarify the efficacy of such a program.

Although there is evidence to support the efficacy of neuraminidase inhibitor prophylaxis, the treatment is expensive (at least $\$ 50$ per day) and was used within 36-48 hours of diagnosis of the index case in the studies reviewed here. Appropriate use during the winter respiratory illness season, when many viruses may be circulating, would require access to rapid microbiologic diagnosis to evaluate suspected exposures, or an active viral surveillance program in the community to determine whether influenza is epidemiologically the most likely cause of respiratory illness in the index case. Health Canada's Fluwatch Program (www.hc-sc.gc.ca/pphb-dgspsp/fluwatch/index.html) provides biweekly summaries of disease activity across Canada, and local laboratories may also provide timely information.

Limitations of our systematic review include the restriction of reviewed publications to French or English and the inability to provide an overall estimate of vaccine efficacy, such as might be obtained through a meta-analysis. However, we concluded that the vaccines and outcome measures of influenza were sufficiently different across trials to prevent pooling of individual trial results.

Evaluation of new influenza vaccines is necessary. A liveattenuated, nasally administered vaccine is now licensed for use in the United States, ${ }^{62}$ and a nasally administered inactivated product is being developed..$^{63}$ Furthermore, determining the efficacy of universal vaccination and treatment programs will require ongoing scrutiny. 
This article has been peer reviewed.

From the Departments of Pediatrics and of Community Health and Epidemiology, Dalhousie University and IWK Health Centre, Halifax, NS (Langley), and the Department of Medicine, Division of Respirology, St. Micheal's Hospital and University of Toronto, Toronto, Ont. (Faughnan)

Competing interests: None declared.

Contributors: Both authors contributed substantially to the conception and design of the systematic review, to the acquisition, analysis and interpretation of data and to drafting the article. They revised it critically for important intellectual content and gave final approval of the version to be published.

\section{References}

1. Glezen WP, Keitel WA, Taber LH, Piedra PA, Clover RD, Couch RB. Age distribution of patients with medically-attended illnesses caused by sequential variants of influenza $\mathrm{A} / \mathrm{H} 1 \mathrm{~N} 1$ : comparison to age-specific infection rates, 1978-1989. Am F Epidemiol 1991;133(3):296-304.

2. Editorial Board Respiratory Disease in Canada. Respiratory disease in Canada. Ottawa: Health Canada;2001.

3. Nichol KL, Margolis KL, Wuorenma J, Von Sternberg T. The efficacy and cost effectiveness of vaccination against influenza among elderly persons living in the community. N Engl 7 Med 1994;331(12):778-84.

4. Fedson DS, Wajda A, Nicol JP, Hammond GW, Kaiser DL, Roos LL. Clinical effectiveness of influenza vaccination in Manitoba. FAMA 1993;270(16) 1956-61.

5. Neuzil KM, Mellen BG, Wright PF, Mitchel EF, Jr., Griffin MR. The effect of influenza on hospitalizations, outpatient visits, and courses of antibiotics in children. N Engl 7 Med 2000;342(4):225-31.

6. Izurieta HS, Thompson WW, Kramarz P, Shay DK, Davis RL, DeStefano F, et al. Influenza and the rates of hospitalization for respiratory disease among infants and young children. NEngl F Med 2000;342(4):232-9.

7. Woolf SH, Battista RN, Anderson GM, Logan AG, Wang E. Assessing the clinical effectiveness of preventive maneuvers: analytic principles and systematic methods in reviewing evidence and developing clinical practice recommendations. A report by the Canadian Task Force on the Periodic Health Examination. 7 Clin Epidemiol 1990;43(9):891-905.

8. Harris RP, Helfand M, Woolf SH, Lohr KN, Mulrow CD, Teutsch SM, et al; Methods Work Group, Third US Preventive Services Task Force. Current methods of the US Preventive Services Task Force: a review of the process. Am 7 Prev Med 2001;20(3 Suppl):21-35.

9. Powers DC, Smith GE, Anderson EL, Kennedy DJ, Hackett CS, Wilkinson $\mathrm{BE}$, et al. Influenza A virus vaccines containing purified recombinant $\mathrm{H} 3$ hemagglutinin are well tolerated and induce protective immune responses in healthy adults. 7 Infect Dis 1995;171(6):1595-9.

10. Edwards KM, Dupont WD, Westrich MK, Plummer WD, Jr., Palmer PS, Wright PF. A randomized controlled trial of cold-adapted and inactivated vaccines for the prevention of influenza A disease. F Infect Dis 1994:169:68-76.

11. Keitel WA, Cate TR, Couch RB. Efficacy of sequential annual vaccination with inactivated influenza virus vaccine. Am 7 Epidemiol 1988;127(2):353-64.

12. Monto AS, Miller FD, Maassab HF. Evaluation of an attenuated, cold-recombinant influenza B virus vaccine. F Infect Dis 1982;145(1):57-64.

13. Hammond ML, Ferris AA, Faine S, McAvan T. Effective protection against influenza after vaccination with subunit vaccine. Med 7 Aust 1978;1(6):301-3.

14. Rytel MW, Jackson LJ, Niebojewski RA, Haagensen JL, Rosenkranz MA. Field trial of live attenuated influenza A/B (“Alice"/R-75) vaccine. Am 7 Epidemiol 1977; 105(1):49-55.

15. Mair HJ, Sansome DA, Tillett HE. A controlled trial of inactivated monovalent influenza A vaccines in general practice. 7 Hyg (Lond) 1974;73(2):317-27.

16. Leibovitz A, Coultrip RL, Kilbourne ED, Legters LJ, Smith CD, Chin J, et al. Correlated studies of a recombinant influenza-virus vaccine. IV. Protection against naturally occurring influenza in military trainees. F Infect Dis 1971;124 (5):481-7.

17. Bridges CB, Thompson WW, Meltzer MI, Reeve GR, Talamonti WJ, Cox NJ, et al. Effectiveness and cost-benefit of influenza vaccination of healthy working adults: A randomized controlled trial. 7AMA 2000;284(13):1655-63.

18. Tannock GA, Bryce DA, Hensley MJ, Saunders NA, Gillett RS, Kennedy WS. Responses to one or two doses of a deoxycholate subunit influenza vaccine in a primed population. Vaccine 1984;2(1):100-6.

19. Mixeu MA, Vespa GN, Forleo-Neto E, Toniolo-Neto J, Alves PM. Impact of influenza vaccination on civilian aircrew illness and absenteeism. Aviat Space Environ Med 2002;73(9):876-80.

20. Nichol KL, Mendelman PM, Mallon KP, Jackson LA, Gorse GJ, Belshe RB, et al. Effectiveness of live, attenuated intranasal influenza virus vaccine in healthy, working adults: a randomized controlled trial. FAMA 1999;282(2): 137-44.

21. Nichol KL, Lind A, Margolis KL, Murdoch M, McFadden R, Hauge M, et al. The effectiveness of vaccination against influenza in healthy, working adults. N Engl 7 Med 1995;333(14):889-93.

22. Williams MC, Davignon L, McDonald JC, Pavilanis PV, Boudreault A, Clayton AJ. Trials of aqueous killed influenza vaccine in Canada, 1968-69. Bull World Health Organ 1973;49(4):333-40.

23. Waldman RH, Coggins WJ. Influenza immunization: field trial on a university campus. F Infect Dis 1972;126(3):242-8.

24. Edmonson K, Graham D, Warburton M. A clinical trial of influenza vaccine in Canberra. Med 7 Australia 1970;4:6-13.

25. Eddy TS, Davies NA. The effect of vaccine on a closed epidemic of Hong Kong influenza. S Afr Med 7 1970;44(8):214-6.

26. Hobson D, Baker FA, Chivers CP, Reed SE, Sharp D. A comparison of monovalent Hong Kong influenza virus vaccine with vaccines containing only pre-1968 Asian strains in adult volunteers. A report to the Medical Research Council Committee on Influenza and other Respiratory Virus Vaccines. 7 Hyg (Lond) 1970;68(3):369-78.

27. Nichol KL, Goodman M. Cost effectiveness of influenza vaccination for healthy persons between ages 65 and 74 years. Vaccine 2002;20(Suppl 2):S21-4.

28. Neuzil KM, Dupont WD, Wright PF, Edwards KM. Efficacy of inactivated and cold-adapted vaccines against influenza A infection, 1985 to 1990: the pediatric experience. Pediatr Infect Dis 7 2001;20(8):733-40.

29. Hurwitz ES, Haber M, Chang A, Shope T, Teo ST, Giesick JS, et al. Studies of the 1996-1997 inactivated influenza vaccine among children attending day care: immunologic response, protection against infection, and clinical effectiveness. F Infect Dis 2000;182(4):1218-21.

30. Belshe RB, Gruber WC, Mendelman PM, Cho I, Reisinger K, Block SL, et al. Efficacy of vaccination with live attenuated, cold-adapted, trivalent, intranasal influenza virus vaccine against a variant (A/Sydney) not contained in the vaccine. 7 Pediatr 2000;136(2):168-75

31. Belshe RB, Mendelman PM, Treanor J, King J, Gruber WC, Piedra P, et al. The efficacy of live attenuated, cold-adapted, trivalent, intranasal influenzavirus vaccine in children. N Engl 7 Med 1998;338(20):1405-12.

32. Gruber WC, Belshe RB, King JC, Treanor JJ, Piedra PA, Wright PF, et al Evaluation of live attenuated influenza vaccines in children 6-18 months of age: safety, immunogenicity, and efficacy. National Institute of Allergy and Infectious Diseases, Vaccine and Treatment Evaluation Program and the Wyeth-Ayerst ca Influenza Vaccine Investigators Group. 7 Infect Dis 1996;173 (6):1313-9.

33. Clover RD, Crawford S, Glezen WP, Taber LH, Matson CC, Couch RB. Comparison of heterotypic protection against influenza A/Taiwan/86 (H1N1) by attenuated and inactivated vaccines to A/Chile/83-like viruses. 7 Infect Dis 1991;163(2):300-4.

34. Gruber WC, Taber LH, Glezen WP, Clover RD, Abell TD, Demmler RW, et al. Live attenuated and inactivated influenza vaccine in school-age children. Am 7 Dis Child 1990;144(5):595-600.

35. Feldman S, Wright PF, Webster RG, Roberson PK, Mahoney J, Thompson $\mathrm{J}$, et al. Use of influenza A virus vaccines in seronegative children: live coldadapted versus inactivated whole virus. 7 Infect Dis 1985;152(6):1212-8.

36. Hoskins TW, Davies JR, Allchin A, Miller CL, Pollock TM. Controlled tria of inactivated influenza vaccine containing the a-Hong Kong strain during an outbreak of influenza due to the a-England-42-72 strain. Lancet 1973;2(7821): $116-20$

37. Wesselius-de Casparis A, Masurel N, Kerrebijn KF. Field trial with human and equine influenza vaccines in children: protection and antibody titres. Bull World Health Organ 1972;46(2):151-7.

38. Colombo C, Argiolas L, La Vecchia C, Negri E, Meloni G, Meloni T. Influenza vaccine in healthy preschool children. Rev Epidemiol Sante Publique 2001 49(2):157-62.

39. Khan AS, Polezhaev F, Vasiljeva R, Drinevsky V, Buffington J, Gary H, et al. Comparison of US inactivated split-virus and Russian live attenuated, coldadapted trivalent influenza vaccines in Russian schoolchildren. 7 Infect Dis 1996;173(2):453-6

40. Rudenko LG, Slepushkin AN, Monto AS, Kendal AP, Grigorieva EP, Burtseva EP, et al. Efficacy of live attenuated and inactivated influenza vaccines in schoolchildren and their unvaccinated contacts in Novgorod, Russia. 7 Infect Dis 1993;168(4):881-7.

41. Alexandrova GI, Budilovsky GN, Koval TA, Polezhaev FI, Garmashova LM, Ghendon YuZ, et al. Study of live recombinant cold-adapted influenza bivalent vaccine of type A for use in children: an epidemiological control trial. Vaccine 1986;4(2):114-8.

42. Maynard JE, Dull HB, Hanson ML, Feltz ET, Berger R, Hammes L. Evaluation of monovalent and polyvalent influenza vaccines during an epidemic of type A2 and B influenza. Am 7 Epidemiol 1968;87(1):148-57.

43. Hayden FG, Treanor JJ, Fritz RS, Lobo M, Betts RF, Miller M, et al. Use of the oral neuraminidase inhibitor oseltamivir in experimental human influenza: randomized controlled trials for prevention and treatment. FAMA 1999;282 (13):1240-6.

44. Hayden FG, Atmar RL, Schilling M, Johnson C, Poretz D, Paar D, et al. Use of the selective oral neuraminidase inhibitor oseltamivir to prevent influenza. New Engl 7 Med 1999;341(18):1336-43.

45. Welliver R, Monto AS, Carewicz O, Schatteman E, Hassman M, Hedrick J, 
et al; Oseltamivir Post Exposure Prophylaxis Investigator Group. Effectiveness of oseltamivir in preventing influenza in household contacts: a randomized controlled trial. 7AMA 2001;285(6):748-54.

46. Kaiser L, Henry D, Flack NP, Keene O, Hayden FG. Short-term treatment with zanamivir to prevent influenza: results of a placebo-controlled study. Clin Infect Dis 2000;30:587-9.

47. Hayden FG, Gubareva LV, Monto AS, Klein TC, Elliot MJ, Hammond JM, et al. Inhaled zanamivir for the prevention of influenza in families. Zanamivir Family Study Group. N Engl 7 Med 2000;343(18):1282-9.

48. Monto AS, Pichichero ME, Blanckenberg SJ, Ruuskanen O, Cooper C, Fleming DM, et al. Zanamivir prophylaxis: an effective strategy for the prevention of influenza types A and B within households. 7 Infect Dis 2002;186 (11):1582-8.

49. Stiver G. The treatment of influenza with antiviral drugs. CMA7 2003;168(1): 49-56.

50. Hilleman MR. Realities and enigmas of human viral influenza: pathogenesis, epidemiology and control. Vaccine 2002;20(25-26):3068-87.

51. Palache A. Influenza subunit vaccine - ten years experience. Eur 7 Clin Research 1992;3:117-38.

52. Squires SG, Macey JF, Tam T. Progress toward Canadian target coverage rates for influenza and pneumococcal immunizations. Can Commun Dis Rep 2001;27(10):90-1.

53. Bridges CB, Harper SA, Fukuda K, Uyeki TM, Cox NJ, Singleton JA. Prevention and control of influenza. Recommendations of the Advisory Committee on Immunization Practices (ACIP). MMWR Recomm Rep 2003;52(RR8):1-34; quiz CE1-4.

54. Simonsen L, Fukuda K, Schonberger LB, Cox NJ. The impact of influenza epidemics on hospitalizations. F Infect Dis 2000;181(3):831-7.

55. Strikas RA, Wallace GS, Myers MG. Influenza pandemic preparedness action plan for the United States: 2002 update. Clin Infect Dis 2002;35(5):590-6.

56. National Advisory Committee on Immunization (NACI). Canadian immunization guide. 6th ed. Ottawa: Canadian Medical Association;2002.

57. Committee on Infectious Diseases American Academy of Pediatrics. Recommendations for influenza immunization of children. Pediatrics 2004;113(5): 1441-7.

58. Harper SA, Fukuda K, Uyeki TM, Cox NJ, Bridges CB; Centers for Disease Control and Prevention (CDC) Advisory Committee on Immunization Practices (ACIP). Prevention and control of influenza: recommendations of the Advisory Committee on Immunization Practices (ACIP) [published erratum in MMWR Recomm Rep 2004;53(32):743]. MMWR Recomm Rep 2004;53(RR6): $1-40$.

59. Demicheli V. Mass influenza vaccination in Ontario: is it worthwhile? CMAf 2001;164(1):38-9; discussion 40-1.

60. Schabas RE. Mass influenza vaccination in Ontario: a sensible move. CMAf 2001;164(1):36-7.

61. Reichert TA, Sugaya N, Fedson DS, Glezen WP, Simonsen L, Tashiro M. The Japanese experience with vaccinating schoolchildren against influenza. $N$ Engl 7 Med 2001;344(12):889-96.

62. Harper SA, Fukuda K, Cox NJ, Bridges CB. Using live, attenuated influenza vaccine for prevention and control of influenza: supplemental recommendations of the Advisory Committee on Immunization Practices (ACIP). $M M W R$ Recomm Rep 2003;52(RR-13):1-8.

63. Haneberg B, Holst J. Can nonliving nasal vaccines be made to work? Expert Rev Vaccines 2002;1(2):227-32.

Correspondence to: Joanne M. Langley, Clinical Trials Research Center, IWK Health Centre, 5850 University Ave., Halifax NS B3J 3G9; fax 902 470-7217; joanne.langley@dal.ca

\section{Appendix 1: Canadian Task Force on Preventive Health Care levels of evidence used to rate research design and quality of individual studies*}

\section{Research design rating}

I Evidence from at least 1 randomized controlled trial

II-1 Evidence from controlled trial(s) without randomization

II-2 Evidence from cohort or case-control analytic studies, preferably from more than 1 centre or research group

II-3 Evidence from comparisons between times or places with or without the intervention; dramatic results from uncontrolled studies could be included here

III Opinions of respected authorities, based on clinical experience; descriptive studies or reports of expert committees

\section{Quality (internal validity) rating†}

Good A study that meets all design-specific criteria well

Fair A study that does not meet (or it is not clear that it meets) at least one design-specific criterion* but has no known "fatal flaw"

Poor A study that has at least 1 design-specific "fatal flaw," or an accumulation of lesser flaws to the extent that the results of the study are not deemed able to inform recommendations

*The task force's methodology is described in Woolf et $\mathrm{al}^{7}$ and is available from the task force's Web site (www.ctfphc.org, click on History and Methods).

†General design-specific criteria by study type are outlined in Harris et al. ${ }^{8}$ 